\title{
Sur des Culicoides (Diptera: Ceratopogonidae) dont une espèce et une variété nouvelles du Midi de la France
}

\author{
Par J. CALlOT, M. KREMER et J.-A. RIOUX
}

\begin{abstract}
Dans cette note, nous signalerons, parfois après d'autres, des espèces du genre Culicoides trouvées dans le Midi de la France, et nous donnerons un aperçu des espèces françaises du groupe « odibilis» (1).
\end{abstract}

\section{I. - Etude morphologique}

CULICOIDES AGATHENSIS n. sp. (fig. 1, 2, 3 et $4 \mathrm{C}, a$ ).

Espèce d'assez grande taille, d'aspect général intermédiaire entre $C$. maritimus et C. pictipennis. Couleur du thorax brun-olive à pruinosité légèrement dorée. Scutum sans dessin, à part les taches réniformes et la fossette préscutale. Scutellum de la même teinte que le scutum ou légèrement plus clair. Abdomen blanchâtre à bandes brunes. Pattes brun clair à genoux noirs entourés d'une zone plus claire. Ailes grises, brunâtres, à taches claires: disposition des taches comme sur la figure 4 C. $a$. Notons que la seconde cellule radiale est en partie comprise dans la tache claire qui lui fait suite. Les taches claires, situées à l'extrémité des cellules $\mathrm{r} 5, \mathrm{~m} 1$ et généralement $\mathrm{m} 2$, sont séparées du bord de l'aile par une étroite bande sombre. La tache claire située à la base de la cellule $\mathrm{m} 1$ s'étend plus distalement que celle située au-dessus d'elle sur le bord antérieur de l'aile. Macrotriches abondantes, longues et couvrant toute la surface de l'aile, y compris la cellule basale.

\section{Mâle :}

Yeux soudés. Longueur des articles du palpe : 76/65,5/30/42 $\mu$.

Longueur des articles antennaires: 122/49/49/46/46/47/46/46/46/55/177/ $129 / 129 \mu$.

Présence de sensilles sur les articles : 3, 10, 13, 14, 15.

(1) Nous remercions M.-C. Paradis, moniteur à la Faculté de Strasbourg pour les mensurations des exemplaires. 
Ailes comme chez la femelle, mais les taches claires plus étendues et plus confluentes.

Hypopygium : lamelle bien fendue. Processus digitiformes et divergents.

Coxite fortement renflé dans sa moitié proximale, légèrement cylindrique dans sa moitié distale (fig. 1).

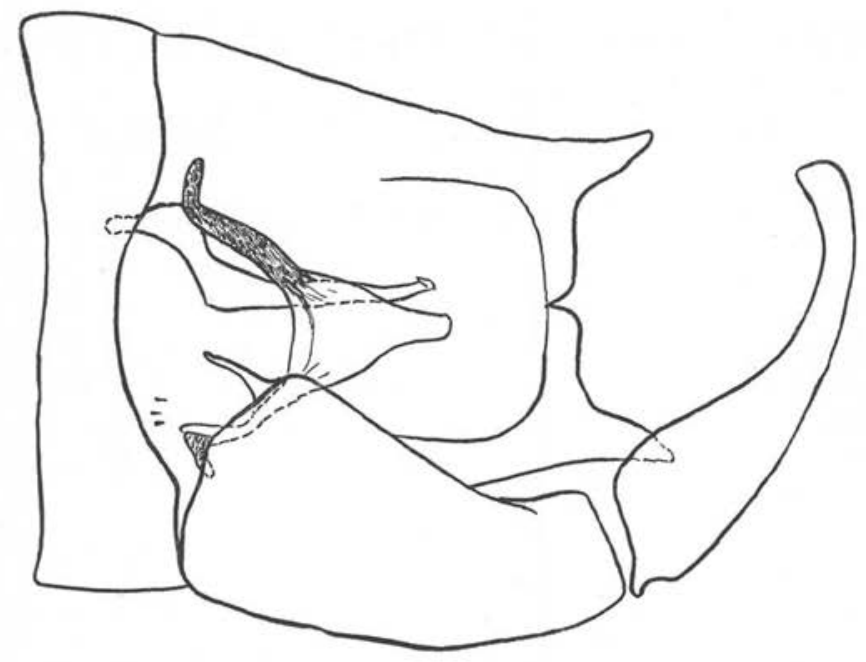

Fig. 1. - Culicoides agathensis $\mathrm{n}$. sp. $\delta^{x}$ : hypopygium

Aedeagus trapézoïdal, la partie distale se termine un peu en rectangle. Bras bien chitinisés et envoyant des prolongements dans le corps. Paramères épaissis et bien chitinisés dans leur partie proximale ; leur partie distale en forme de lame plate ; l'extrémité n'est pas effilée. Sternite échancré, en pentes douces. Membrane basale nue (ou présentant, très rarement, un ou deux spicules).

\section{Femelle :}

Yeux séparés, réunis au-dessus du poil médian par une suture brisée, cette suture se prolongeant généralement sur le vertex par une suture sagittale (fig. 2).

Longueur des articles du palpe : 92/101/33/39 $\mu$ (les deux premiers articles comptés ensemble). Troisième article renflé en triangle. Fossette sensorielle large, modérément profonde, remplie de sensilles.

Longueur des articles des antennes: 56/38/38/40/39/39/40/44/83/79/87/ $94 / 129 \mu$.

Répartition des sensilles sur les articles $3-15: 6,4 / 1,2 / 1 / 0,9 / 1 / 1 / 1,2 / 1,3 / 1 / 1 / 1 /$ 4,1/1. Leur nombre total variant de 19-26. L'indice antennaire varie entre 1,34 et 1,47. 
Les articles proximaux sont en forme de tonneaux; les cinq derniers, allongés, sont plus ou moins cylindriques.

Ailes: longueur comprise entre 1,58 et $1,70 \mathrm{~mm}$ (fig. 4, C, a).

Peigne tibial postérieur à cinq épines, dont les longueurs moyennes sont : 64/69/ $56 / 49 /(49) \mu$; la cinquième soie est très souvent absente.

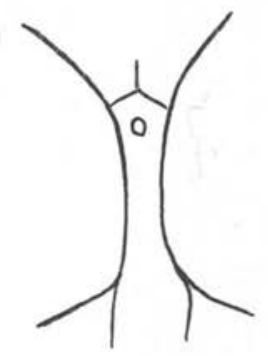

Fig. 2. - C. agathensis $\uparrow$ : espace interoculaire.

Deux spermathèques fonctionnelles de 62 et $55 \mu$ en moyenne ; une spermathèque rudimentaire.

Les types et cotypes, trouvés à Roque-Haute, Portiragne, Hérault, sont déposés à l'Institut de Parasitologie de Strasbourg.
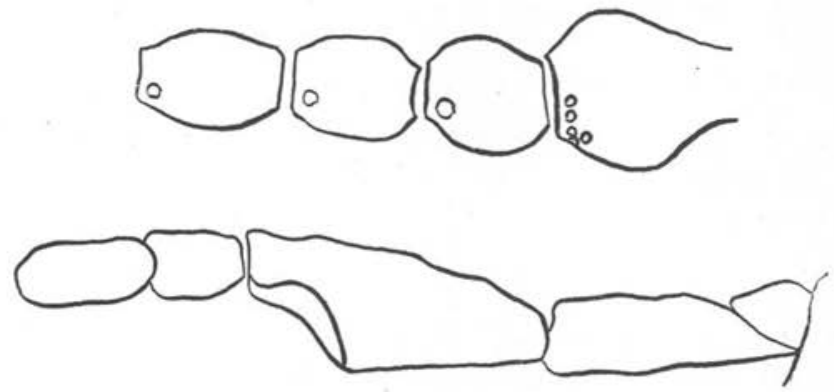

Fig. 3. $-C$. agathensis $q$ : palpe et base du flagellum

\section{CULICOIDES MARITIMUS PAUCISENSILLATUS n. var.}

Les espèces du groupe « odibilis » ont par définition un sensille sur le dernier article de l'antenne. Or, parmi les Culicoides maritimus que nous avons recueillis dans le Midi, nous avons été frappés par le nombre d'exemplaires femelles qui ne possédaient aucun sensille sur le $15^{\circ}$ article. Les mâles de cette variété n'ont pu être séparés, ils sont absolument identiques à ceux du type de $C$. maritimus. Il n'y a pas non plus de différences dans la morphologie, en dehors de cette absence de sensille, entre le type et la variété. 


\section{Femelles :}

Yeux séparés, réunis au-dessus du poil médian par une suture brisée, souvent prolongée sur le vertex par une suture sagittale

Longueur moyenne des articles du palpe : 83/82/36/36 $\mu$.

Longueur moyenne du palpe : $236 \mu$ (max. : 276 ; min. : 216).

Longueur des articles des antennes: 49/31/32/33/35/35/37/38/64/65/75/ $78 / 105 \mu$.

Longueur moyenne de l'antenne : $678 \mu$ (max. : 775 ; min. : 642).

Indice antennaire compris entre 1,23 et 1,45 .

Répartition des sensilles sur les articles 3-15 : 5,3/0,9/1,1/1,1/1,2/0,75/1,15/1,05/ $0,8 / 0,75 / 1 / 3,8 / 0$. Nombre total variant de $14-28,5$.

Longueur des ailes comprise entre 1,24 et $1,51 \mathrm{~mm}$.

Peigne tibial de 4-5 dents, dont les longueurs moyennes sont : 59/62/53/48/(57) $\mu$; la cinquième est exceptionnelle.

Notons que, chez le Culicoides maritimus typique, le nombre total des sensilles est compris entre 29 et 36 et qu'il y en a toujours sur le $15^{\circ}$ article. Les autres mensurations sont autrement identiques pour le type et la variété; le type semble être un peu plus grand, mais cette différence de taille n'est pas statistiquement valable.

Ces exemplaires typiques et la variété ont été trouvés à plusieurs reprises dans les mêmes gîtes.

Les types de la variété, déposés à l'Institut de Parasitologie de Strasbourg, proviennent de St-Pierre-sur-Mer (Aude).

CULICOIDES CATANEII Clastrier, 1957.

Les exemplaires que nous avons obtenus sont absolument conformes à la description originale. Cependant, le thorax, sur les exemplaires examinés frais, présente un petit triangle sombre sur les côtés.

\section{Femelles:}

Longueur du palpe comprise entre 192 et $213 \mu$. Longueur moyenne des articles : $69 / 76 / 27 / 29 \mu$ (les deux premiers comptés ensemble).

Longueur des antennes comprise entre 568 et $638 \mu$. Longueur moyenne des articles: $42 / 29 / 30 / 30 / 31 / 31 / 32 / 32 / 59 / 60 / 69 / 69104 \mu$. Indice antennaire entre 1,37 et 1,45 .

Répartition des sensilles sur les articles 3-15: 5,9/1,3/1,6/1/1/0,9/1,1/0/1/1/ $1,1 / 2 / 1$.

Peigne tibial à quatre dents de 54/49/43/40 $\mu$.

CULICOIDES DUDDINGSTONI Kettle et Lawson.

Un mâle et une femelle ont été obtenus d'un prélèvement effectué dans une rizière à Roque-Haute (commune de Portiragne, Hérault). 
Thorax et ailes, génitalia conformes à la description originale. Il faut cependant noter que, sur l'exemplaire mâle, l'extrémité effilée des paramères était à peu près droite. La membrane n'était pas spiculée. Cette absence de spicules a, du reste, été signalée depuis la description originale, et nous-mêmes, comme Campbell et Pelham-Clinton, avons souvent vu des exemplaires avec seulement de très rares spicules. Il y a aussi sur nos exemplaires méridionaux, chez la femelle, moins de sensilles qu'en Grande-Bretagne, mais la répartition en est la même.

\section{II. - Discussion systématique}

Rappelons qu'on entend généralement par groupe « odibilis » des espèces ayant : des ailes grises à taches claires; la deuxième cellule radiale (sauf exception) entièrement sombre ; un thorax le plus souvent sans taches. L'hypopygium mâle a des apodèmes ventraux simples, une lamelle fendue, un ædeagus plus ou moins triangulaire ou trapézoïdal, une membrane nue ou spiculée. Les femelles ont les yeux séparés, des sensilles sur tous les articles du palpe (avec exceptions) et deux spermathèques.

Nous avons proposé de scinder ce groupe en deux sous-groupes: le sous-groupe « simulator », chez lequel il n'y a pas de taches claires à la base de la cellule $\mathrm{m} 1$, et le sous-groupe " odibilis» sensu stricto, ayant une tache dans la base de $\mathrm{m} 1$.

Rappelons que les espèces françaises du sous-groupe «simulator » sont : C. begueti. C. haranti, C. musilator et $C$. cubitalis (dans la mesure où cette espèce, dépourvue de taches alaires distales, peut être incluse dans ce sous-groupe).

Dans le sous-groupe « odibilis », au sens strict, nous plaçons : C. odibilis, C. pictipennis, C. duddingstoni, C. maritimus, C. maritimus paucisensillatus, C. agathensis, C. clastrieri, C. cataneii.

Nous avons donc trouvé dans le Midi les espèces suivantes: $C$. duddingstoni, C. maritimus et sa variété, $C$. agathensis. Harant y avait antérieurement signalé $C$. pictipennis.

La diagnose de $C$. maritimus ne pose pas de difficultés spéciales.

Le mâle possède une membrane abondamment spiculée, ce qui constitue une exception dans ce groupe. Les ailes sont caractéristiques: la deuxième cellule radiale est sombre, les taches de l'extrémité des cellules $\mathrm{r} 5$ et $\mathrm{m} 1$ sont nettement séparées du bord de l'aile. La tache claire de la base de $\mathrm{m} 1$ s'étend plus distalement que celle placée en avant d'elle.

Les femelles, dans le type, ont des sensilles sur le $15^{\circ}$ article de l'antenne. Ils manquent sur cet article dans la variété signalée plus haut.

L'hypopygium des exemplaires de $C$. cataneii est typique : ædeagus large, presque rectangulaire ; paramères rapidement et fortement effilés, à extrémité pratiquement rectiligne ; apodème ventral long, assez large, contourné. Deuxième cellule radiale sombre; taches de l'extrémité des cellules $\mathrm{r} 5$ et $\mathrm{ml}$ nettement séparées du bord de l'aile; la tache située à la base de la cellule $\mathrm{m} 1$ ne s'étend pas plus loin que celle située audessus d'elle. 

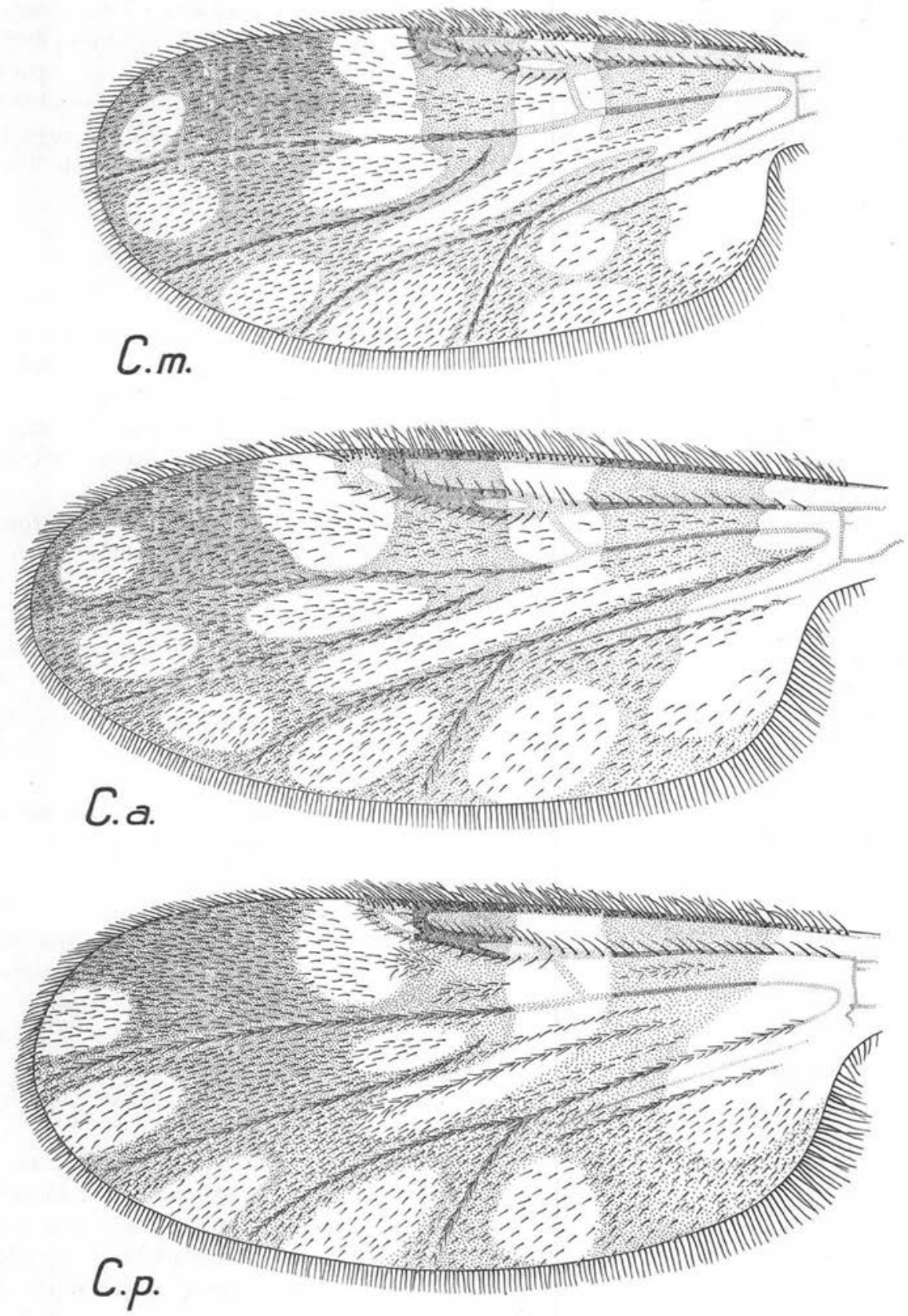

FIG. 4. - Ailes des femelles. C. m.: C. maritimus; C. a. : C. agathensis; C. p.: C. pictipennis 
Peu de sensilles sur les antennes des femelles; il n'y en a jamais sur le $10^{\circ}$ article.

Rappelons que $C$. pictipennis a une deuxième cellule radiale entamée en grande partie par une tache claire ; que la tache située à la base de la cellule $\mathrm{m} 1$ ne s'étend pas plus distalement que cette tache. Le bord de l'aile coupe les taches situées à l'extrémité des cellules $\mathrm{r} 5$ et $\mathrm{ml}$ (fig. $4, \mathrm{C}, a$ ). Les femelles possèdent des sensilles sur tous les articles et l'indice antennaire est supérieur à 1,55 .

C. agathensis présente une seconde cellule radiale entamée en partie par la tache claire qui lui fait suite, comme chez $C$. pictipennis; par contre, les taches situées à l'extrémité des cellules $\mathrm{r} 5$ et $\mathrm{m} 1$ sont séparées du bord de l'aile par une zone sombre (fig. $4, \mathrm{C}, a$ ), et la tache située à la base de $\mathrm{ml}$ se prolonge plus distalement que celle située au-dessus d'elle.

Ces caractères permettent de distinguer les trois espèces : $C$. maritimus, $C$. pictipennis, C. agathensis. Chez ce dernier, la membrane de l'ædeagus est nue, comme chez C. pictipennis, mais on peut cependant trouver sur quelques exemplaires deux à trois spicules en ligne sur cette membrane.

L'indice antennaire de $C$. agathensis est proche de celui des femelles de $C$. maritimus. La disposition générale des sensilles est la même dans les deux espèces, mais leur nombre total est toujours moindre chez $C$. agathensis que chez $C$. maritimus type.

Les hypopygiums mâles de $C$. agathensis et de $C$. pictipennis sont très proches; cependant, chez agathensis, la base du coxite et le corps de l'ædeagus sont plus larges.

Les descriptions ci-dessus peuvent faire douter de la valeur de la disposition des sensilles antennaires dans la systématique des Culicoides et dans leur groupement, d'autant plus que nous avons observé, sur un exemplaire de chacune des trois espèces que nous venons d'étudier, $C$. maritimus, $C$. agathensis et $C$. pictipennis, la présence d'un sensille sur le $15^{\circ}$ article d'un côté et son absence de l'autre...

La diagnose de $C$. duddingstoni, seule espèce du groupe « odibilis » à posséder un thorax orné de taches punctiformes, ne présente pas de difficultés. Ses ailes sont très proches de celles du $C$. odibilis; la seconde cellule radiale est sombre; les taches de l'extrémité touchent le bord de l'aile.

\section{III. - Localisation des espèces trouvées}

Outre les espèces signalées ci-dessus, nous signalons quelques espèces trouvées dans la même prospection; les prélèvements ont été effectués le 4 et le 5 novembre 1961. Les adultes ont été obtenus au laboratoire.

$1^{\circ}$ Culicoides punctatus Meig. Mare creusée dans du basalte. Typhaie dulçaquicole, Roque-Haute, commune de Portiragne (Hérault).

$2^{\circ}$ Culicoides circumscriptus Kieff. Puits à l'Espiguette, Grau-du-Roi (Gard), et roubine à Phagmites, même endroit. Bras temporaire du Vidoule, Grau-du-Roi, sables légèrement salés. Roubine à Salicornia fruticosa et canal de drainage, Sylvéréal, Petite-Camargue (Gard).

Mare à Salins-de-Badon, Camargue (B.-du-R.), phragmitaie semi-halophile. 


\begin{tabular}{|c|c|c|c|c|c|c|c|c|}
\hline 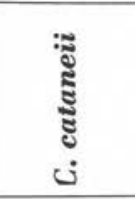 & 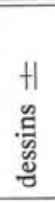 & & $\begin{array}{l}0 \\
\text { हू } \\
\text { है }\end{array}$ & 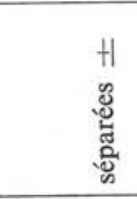 & 己 & 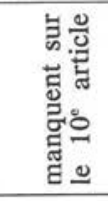 & 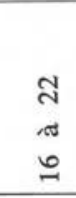 & $\begin{array}{l}\text { a } \\
\text { ñ } \\
\text { ñ }\end{array}$ \\
\hline 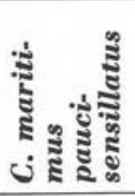 & 0 & & $\begin{array}{l}0 \\
\text { हू } \\
\text { है }\end{array}$ & 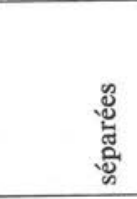 & $\frac{8}{\frac{0}{3}}$ & 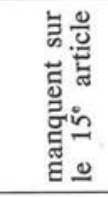 & $\begin{array}{l}\text { ปे } \\
\text { む } \\
\pm\end{array}$ & 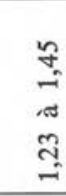 \\
\hline है & 0 & & 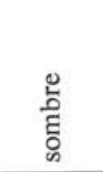 & 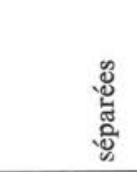 & $\frac{\mathscr{d}}{3}$ & 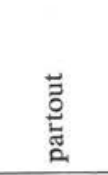 & $\begin{array}{l}\text { D } \\
\text { సे }\end{array}$ & $\begin{array}{l}\text { m } \\
\text { i } \\
0 \\
\text { m }\end{array}$ \\
\hline 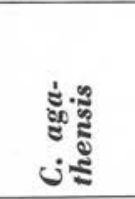 & 0 & & 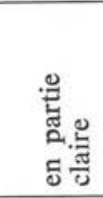 & 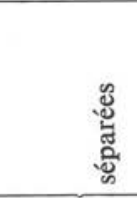 & 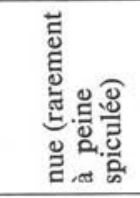 & 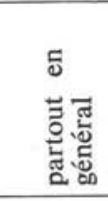 & $\begin{array}{l}\text { i } \\
\text { i } \\
2\end{array}$ & 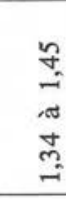 \\
\hline 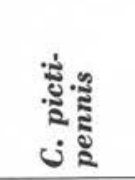 & 0 & & 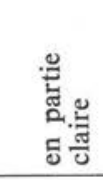 & 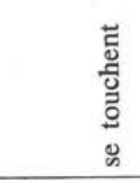 & き & 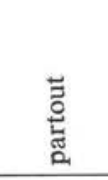 & $\begin{array}{l}\text { లే } \\
\text { స్ } \\
\text { స }\end{array}$ & 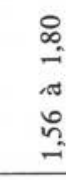 \\
\hline 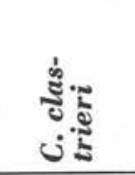 & 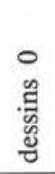 & & $\begin{array}{l}0 \\
\text { हू } \\
\text { हू }\end{array}$ & 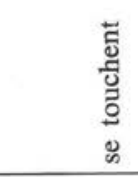 & $\stackrel{\Xi}{\Xi}$ & 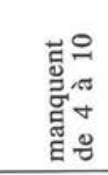 & $\begin{array}{l}\tilde{N} \\
\approx \\
\beth\end{array}$ & $\begin{array}{l}\text { ñ } \\
\text { జ } \\
\text { mे }\end{array}$ \\
\hline 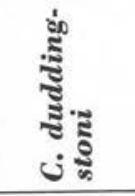 & $\begin{array}{l}+ \\
\text {. } \\
\text { 离 } \\
\text { वे }\end{array}$ & 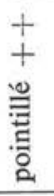 & $\begin{array}{l}0 \\
\text { है } \\
0\end{array}$ & 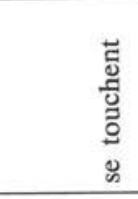 & 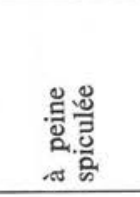 & 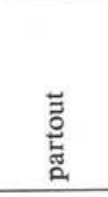 & $\begin{array}{l}\text { J } \\
i \\
\dot{q}\end{array}$ & 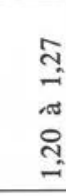 \\
\hline$\frac{\substack{\pi \\
: \frac{\pi}{\pi}}}{0}$ & $\begin{array}{l}\mathscr{H} \\
\text { है } \\
\text { वे }\end{array}$ & $\begin{array}{l}+ \\
+ \\
+ \\
+\end{array}$ & Еั & 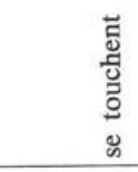 & $\stackrel{\Xi}{\Xi}$ & 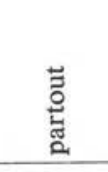 & $\begin{array}{l}0 \\
\pi \\
\pi\end{array}$ & $\begin{array}{l}n \\
\approx \\
\approx \\
=\end{array}$ \\
\hline & 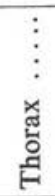 & & 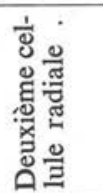 & 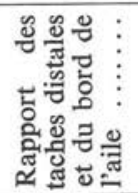 & 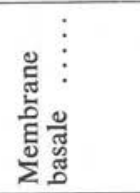 & 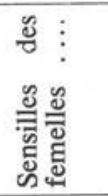 & 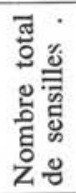 & 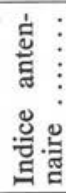 \\
\hline
\end{tabular}


Divers points plus ou moins salés : pont des Tourradons, Le Cailar (Gard).

Mares artificielles et différents terrains marécageux salés ; Saint-Pierre-la-Mer (Aude).

Cette espèce a déjà été signalée par Harant et Descous (1959) à Montpellier.

$3^{\circ}$ Culicoides cataneii Clastrier. Espiguette, puits et roubine comme plus haut. Rizière non moissonnée, Portiragne (Hérault).

Mare à Typha, Roque-Haute, Portiragne.

$4^{\circ}$ Culicoides agathensis n. sp. Mare à Typha, Roque-Haute, Portiragne.

Mares salées, Saint-Pierre-la-Mer (Aude).

$5^{\circ}$ Culicoides maritimus Kieff (1). Mare à Phragmites, Salins-de-Badon, en Camargue.

Mares le long du canal du Rhône à Sète, Le Cailar (Gard).

Mares variées, Saint-Pierre-la-Mer (Aude).

$6^{\circ}$ Culicoides maritimus paucisensillatus n. var. Mêmes gîtes que le précédent et, en plus, roubines et fossés à Sylvéréal (Gard).

$7^{\circ}$ Culicoides duddingstoni Kettle et Lawson. Rizière à Portiragne (Hérault). Un mâle éclos le 8 mai 1962.

\section{Résumé}

Nous signalons la présence, ou nous décrivons, de Camargue et de divers points du Languedoc, de Culicoides punctatus Meig., C. circumscriptus Kieff., C. cataneii Clastrier, $C$. duddingstoni Kettle et Lawson, C. maritimus Kieff., C. maritimus paucisensillatus nov. var., $C$. agathensis n. sp.

Nous revoyons, à propos de ces cinq dernières espèces, les caractères du groupe * odibilis » sensu stricto.

\section{Bibliographie}

Campbell (J. A.) et Pelham-Clinton (E. C.) (1960). - A taxonomic review of the British species of Culicoides Latreille (Diptera: Ceratopogonidae). Proc. R. Soc. Edinburgh (B.), 68, 181-302.

Clastrier (J.) (1957). - Notes sur les Cératopogonidés. II. Sur quelques Culicoides d'Algérie à ailes tachetées. Arch. Inst. Pasteur Algérie, 35, 404-444.

EdWARDS (F. W.) (1939). - Ceratopogonidae, in Edwards, Oldroyd et Smart. British bloodsucking flies, British Museum, Londres.

Harant (H.) et Descous (S.) (1959). - Un cératopogonidé nouveau pour la faune de France: Culicoides circumscriptus Kieffer 1918. Cahier Natur., n.s., 15, 1-2.

Kettue (D. S.) et Lawson (J.H.W.) (1955). - Description of two species of Culicoides Latreille (Diptera: Ceratopogonidae) new to science. Proc. R. Entomol. Soc. London (B.), 24, 37-47.

Kremer (M.) et Callot (J.) (1961). - Culicoides musilator n. sp. et espèces du groupe " odibilis » nouvelles pour la faune française (Diptera: Ceratopogonidae). Ann. Parasitol., 36, 689-699.

Rioux (J.-A.), Descous (S.) et PeCH (J.) (1959). - Un nouveau Cératopogonidé arboricole, Culicoides haranti n. sp. (Diptera: Heleidae). Ann. Parasitol., 34, 432-448.

(Laboratoires de Parasitologie des Facultés de Médecine de Strasbourg et de Montpellier)

(1) En tenant compte que des femelles. 